\title{
Inhibitory effect of soluble EP2 receptor on ovarian tumor growth in nude mice and utility of TMPRSS4 as a combinatorial molecular target
}

\author{
TETSUYUKI TAKAHASHI* ${ }^{*}$, HISANORI UEHARA * and KEISUKE IZUMI \\ Department of Molecular and Environmental Pathology, Institute of Health Biosciences, \\ University of Tokushima Graduate School, Tokushima 770-8503, Japan
}

Received January 25, 2013; Accepted March 15, 2013

DOI: $10.3892 /$ ijo.2013.1957

\begin{abstract}
We have previously reported that FuEP2/Ex2, a soluble decoy receptor for $\mathrm{PGE}_{2}$, suppresses tumor growth in an orthotopic xenograft model. To examine whether it has further uses, we examined the effect of FuEP2/Ex2 in an intraperitoneal metastasis model of ovarian cancer cells. We established FuEP2/Ex2-expressing ovarian cancer cells (SKOV/ip-FuEP2/Ex2) and injected them intraperitoneally into female nude mice. Mice injected with SKOV/ip-FuEP2/Ex2 had no ascitic fluid and showed smaller tumor lesions compared to mice injected with vector control cells, with decreased microvessel density and M2 macrophages. To identify molecular targets for combination treatment, we conducted cDNA microarray analysis and found three genes encoding enzyme [matrix metalloproteinase-7 (MMP-7), transmembrane protease serin 4 (TMPRSS4) and cytocrome P450 1B1 (CYP1B1)] to be upregulated in SKOV/ip-FuEP2/Ex2-derived tumors. Administration of TMPRSS4 inhibitor further reduced tumor weight and decreased the number of Ki-67-positive cells in SKOV/ip-FuEP2/Ex2-injected mice. These data indicate a possible EP-targeting strategy using FuEP2/Ex2 in the treatment of ovarian cancer and suggest that dual targeting of EP-mediated signaling and TMPRSS4 may enhance therapeutic value.
\end{abstract}

\section{Introduction}

Of the gynecologic malignancies, ovarian cancer is the leading cause of death, with an overall 5-year survival rate of

Correspondence to: Dr Tetsuyuki Takahashi, Department of Molecular and Environmental Pathology, Institute of Health Biosciences, University of Tokushima Graduate School, 3-18-15 Kuramoto-cho, Tokushima 770-8503, Japan

E-mail: tetakaha@basic.med.tokushima-u.ac.jp

*Contributed equally

Key words: ovarian cancer, soluble EP2 receptor, decoy receptor, transmembrane protease serin 4 less than $50 \%$. Worldwide in 2008 , approximately 225,500 new cases were diagnosed and there were approximately 140,200 ovarian cancer deaths (1). The main reason for this is the symptoms, which are subjective and ill-defined, making early diagnosis difficult. Ovarian cancer tends to progress very quickly and metastasize into the peritoneal cavity, features that also contribute to its poor prognosis. Generally, current management of ovarian cancer is cytoreductive surgery followed by chemotherapy with paclitaxel and a platinum analog in combination. Although this management induces a favorable outcome, the long-term survival of ovarian cancer patients remains unsatisfactory (2) and efforts to find a novel, effective treatment strategy are ongoing.

Arachidonic acid-derived biolipids, including prostaglandins, prostacyclins, thromboxanes, and leukotrienes play an important role in regulating various biological processes. They are generated by the cyclooxygenase (COX) or lipoxygenase enzymes. Prostaglandin $\mathrm{E}_{2}\left(\mathrm{PGE}_{2}\right)$ is closely associated with cancer cell growth, tumor development and metastasis (3). Level of $\mathrm{PGE}_{2}$ and expression of COX are upregulated in animal models and clinical specimens of ovarian cancer (4-6). $\mathrm{PGE}_{2}$ acts by binding to specific cell-surface receptors called E-prostanoid receptors (EPs). There are four subtypes of EP (EP1-EP4), which can be classified into three types based on their signaling features (7). Consistent with the expression profiles of $\mathrm{PGE}_{2}$ and $\mathrm{COX}$, increases in EP expression or EP-mediated cellular signaling are observed in ovarian cancer lesions (8). These reports suggest that $\mathrm{PGE}_{2}$-mediated EP signaling is a potential target in the treatment of ovarian cancer.

We reported previously that the soluble fragment of human EP2, which contains second extracellular loops (FuEP2/Ex2), can bind to $\mathrm{PGE}_{2}$, inhibits $\mathrm{PGE}_{2}$-induced cellular signaling, and suppresses osteolytic prostate tumor growth and endometrial tumor growth $(9,10)$. Here, we examine the effect of FuEP2/Ex2 in a peritoneal metastasis model of ovarian cancer to determine which genes exhibit altered expression levels in the tumor lesions. Moreover, the inhibitors and specific siRNA of these genes were used to investigate whether they can act in synergy with FuEP2/ Ex2. 


\section{Materials and methods}

Cells, animals and in vivo selection. Human ovarian adenocarcinoma SKOV-3 cells were purchased from European Collection of Cell Cultures (Salisbury, UK). This cell line was maintained in DMEM/F-12 supplemented with $10 \% \mathrm{FBS}, 100 \mathrm{U} / \mathrm{ml}$ penicillin $\mathrm{G}$, and $0.1 \mathrm{mg} / \mathrm{ml}$ streptomycin sulfate. Five-week-old female nude mice were purchased from Charles River Japan (Yokohama, Japan) and housed under specific pathogen-free conditions. Experimental protocols were carried out according to the Guidelines for the Care and Use of Laboratory Animals of the University of Tokushima School of Medicine and approved by the Animal Care and Use Committee.

To obtain a subclone that prefers to grow intraperitoneally, $2 \times 10^{6}$ SKOV-3 cells were injected into the peritoneal cavity of a female nude mouse. Tumor lesions were separated after 12 weeks and minced with a surgical scalpel. The resulting cell suspension was reseeded and cultured for 1 week. Culture was continued until passage seven. This selected subclone was designed as SKOV/ip.

Stable transfection and expression check. We used the mammalian expression vector encoding FuEP2/Ex2 cDNA, known as pFUSE-FuEP2/Ex2 (9). The mock control vector (pFUSE-hFc2, Invivogen, San Diego, CA) or pFUSE-FuEP2/Ex2 (10 $\mu \mathrm{g}$ per $4 \times 10^{5}$ cells) was transfected using a TransFast ${ }^{\mathrm{TM}}$ transfection reagent (Promega, Madison, WI) according to the manufacturer's protocol. Transfected cells were treated with $0.5 \mathrm{mg} / \mathrm{ml}$ zeocin (Invitrogen, Carlsbad, CA) for 2 weeks, with zeocin exposure repeated every 3 days. After selection, the stable transfectants obtained were maintained in complete culture medium containing $0.2 \mathrm{mg} / \mathrm{ml}$ zeocin. These transfectants were designated as SKOV/ip-Fumock and SKOV/ip-FuEP2/Ex2, respectively.

Total RNAs from transfectants were isolated using an RNeasy Mini kit (Qiagen, Valencia, CA). Aliquots of these total RNAs (1 $\mu \mathrm{g} / \mathrm{sample})$ were subjected to RT-PCR under conditions and procedures reported previously (9). The amplicons were as follows: FuEP2/Ex2-hIgG (covers from 5' end of FuEP2/Ex 2 cDNA to 3 ' end of hIgG Fc sequence in expression vector), IL2ss-hIgG (covers from 5' end of IL2 signal sequence in expression vector to 3 ' end of $\mathrm{hIgG} \mathrm{Fc}$ sequence in expression vector), hEP1, hEP2, hEP3, hEP4, hCOX-1, hCOX-2, hmPGES, hcPGES and $\beta$-actin. As to the expression of hCOX-1 and hCOX-2 mRNA, quantitative real-time RT-PCR (qRT-PCR) analysis by the $\Delta \Delta \mathrm{Ct}$ method was also conducted. The condition and procedure have been described previously (10). The primer sequences used in this study are listed in Table I and $\beta$-actin was amplified as an internal standard. Culture media (CM) from parental SKOV/ip, SKOV/ip-Fumock and SKOV/ip-FuEP2/Ex2 cells were also examined for secreted hIgG Fc-fusion proteins derived from the expression vectors using an hIgG ELISA quantification kit (Bethyl Laboratories, Montgomery, TX).

Proliferation assay.SKOV/ip-FumockandSKOV/ip-FuEP2/Ex2 cells $\left(5 \times 10^{3}\right.$ cells/well) were plated on 96 -well microplates and preincubated overnight at $37^{\circ} \mathrm{C}$. Next, the $\mathrm{CM}$ was replaced with $100 \mu \mathrm{l}$ fresh complete medium; after incubation for 24,48 and $72 \mathrm{~h}$, the viable cells were counted by the MTT [3-(4,5-dimethylthiazol-2-yl)-2,5-diphenyltetrazolium bromide] method.
Peritoneal metastasis model in nude mice. SKOV/ip-Fumock or SKOV/ip-FuEP2/Ex 2 cells $\left(2 \times 10^{6}\right.$ cells/mouse) were injected into the peritoneal cavity of nude mice $(n=11$ in the SKOV/ip-Fumock group and $n=10$ in the SKOV/ip-FuEP2/Ex2 group) using a 30 -gauge syringe. From this time, mice were weighed weekly. After 4 weeks of observation, mice were sacrificed after collecting and measuring the volume of hemorrhagic ascites. All disseminated tumors in the peritoneal cavity were collected, total weight of tumors recorded, then a piece of tumor was selected for biological experiment and fixed in $10 \%$ phosphate-buffered formaldehyde for histological analyses. Five tumors in each group were also embedded in Tissue-Tek OCT Compound (Sakura Finetek, Torrance, CA), immediately frozen using liquid nitrogen and stored at $-80^{\circ} \mathrm{C}$. This experiment was repeated twice.

ELISA for VEGF, CXCL1, IL-6 and IL-8. CM (4x10 cells/1 ml of complete medium/well for 48-h incubation) of SKOV/ip-Fumock or SKOV/ip-FuEP2/Ex2 cells was subjected to ELISA for human VEGF (R\&D Systems, Minneapolis, MN), CXCL1 (Abnova, Taipei City, Taiwan), IL-6 and IL-8 (Gen-Probe, San Diego, CA), according to the manufacturer's protocol.

Histological analyses. The formaldehyde-fixed tissues were embedded in paraffin and sectioned at 4-6 $\mu \mathrm{m}$. All sections were subjected to $\mathrm{H} \& \mathrm{E}$ staining. Expression of hIgG Fc and Ki-67 were detected by immunohistochemical staining and apoptotic cells were detected by the terminal deoxynucleotidyltransferase-mediated dUTP nick end-labeling (TUNEL) assay. The frozen tissues were also sectioned at 4-6 $\mu \mathrm{m}$. These frozen sections were subjected to immunohistochemical staining for mouse CD31, CD68 and CD163.

To perform quantitative analysis, the number of Ki-67, CD31, CD68 and CD163-positive cells and TUNEL-positive cells per field were counted microscopically. Polyclonal anti-hIgG Fc antibody (Bethyl Laboratories), anti-Ki-67 antibody (Dako, Glostrup, Denmark), monoclonal anti-mouse CD31 (MEC 13.3, BD Pharmingen, San Jose, CA), monoclonal anti-mouse CD68 (FA-11, AbD Serotec, Oxford, UK), and polyclonal anti-mouse CD163 (M-96, Santa Cruz Biotechnology, Santa Cruz, CA) were used as the primary antibodies at dilutions of 1:200, 1:50, 1:50, 1:100 and 1:50, respectively. Before probing with the primary antibodies, sections were blocked with $1 \%$ hydrogen peroxide in $50 \%$ methanol. The paraffin sections were retrieved by autoclaving in $0.01 \mathrm{M}$ citrate buffer ( $\mathrm{pH}$ 6.0) for $10 \mathrm{~min}$. Visualization was completed using a ChemMate Envision kit/horseradish peroxidase (for anti-hIgG Fc and anti-Ki-67, Dako), goat anti-rat IgG-HRP (for anti-mouse CD31, Jackson ImmunoResearch Laboratories, West Grove, PA)/3,3'-diaminobenzidine and goat anti-rabbit IgG-HRP (for anti-mouse CD163, Jackson ImmunoResearch Laboratories)/3,3'-diaminobenzidine, respectively. In the TUNEL assay, the DeadEnd Colorimetric TUNEL system (Promega) was employed, following manufacturer's protocol. All sections were counterstained with Mayer's hematoxylin (Muto Pure Chemicals, Tokyo, Japan).

cDNA microarray. Total RNAs from SKOV/ip-Fumock- and SKOV/ip-FuEP2/Ex2-derived tumors were isolated using an 
Table I. Primer sequences for semiquantitative and quantitative RT-PCR.

\begin{tabular}{lll}
\hline Target & \multicolumn{1}{c}{ Forward $\left(5^{\prime} \rightarrow 3^{\prime}\right)$} & \multicolumn{1}{c}{ Reverse $\left(5^{\prime} \rightarrow 3^{\prime}\right)$} \\
\hline FuEP2/Ex2-hIgG & GAATTCGCAGTACGTCCAGTACTGC & TCATTTACCCGGAGACAG \\
hIL2ss-hIgG & ATGTACAGGATGCAACTC & TCATTTACCCGGAGACAG \\
EP1 & CCATGGTTATGAGCCCTTGCGGGC & AGATCTTTAGAAGTGGCTGAGGC \\
EP2 & GAATTCG ATGGGCAATGCCTCCAATG & AGATCTAAGGTCAGCCTGTTACTGGC \\
EP3 & GAATTCGATGAAGGAGACCCGGGGCTAC & GAATTCCTACTTGGGAGGCTGAG \\
EP4 & CCATGGTTATGTCCACTCCCGGGGTC & AGATCTTTATATACATTTTCTG \\
COX-1 & GCCGGAGTCTCTTGCTCCGG & GGGCTGGGCCGCAGTGAATT \\
COX-1 (quantitative) & GAGCTATGGCCCAGAAACAACAG & GCATTCAGCAGAGGCCAGAAG \\
COX-2 & CGCAGTACAGAAAGTATC & CTCTGGATCTGGAACAC \\
COX-2 (quantitative) & CCTGAATGTGCCATAAGACTGAC & AAACCCACAGTGCTTGACACAGA \\
mPGES & TACCCCTTCCTTTCCTGGG & CCAGGTATAGCCACGGCG \\
cPGES & TTGCACGCACGCACGTTCAT & CCGGTGGCGACTCCGCTTTT \\
MMP-7 & CGGCAGCTATGCGACTCACC & TGAGCCAGCGTGTTTCCTGG \\
TMPRS4 & GGCTACAGGGAGACCGGGAG & TGAAGCAGTGGGCTGCCGTG \\
CYP1B1 & ACTGATCGGAAACGCGGCGG & ACGGAACTCGGGGTCGTCGT \\
$\beta$-actin & TACAATGAGCTGCGTGTGG & AGATGGGCACAGTGTGGG \\
$\beta$-actin (quantitative) & TGGCACCCAGCACAATGAA & CTAAGTCATAGTCCGCCTAGAAGCA \\
\hline
\end{tabular}

RNeasy Mini kit (Qiagen), then used to perform cDNA microarray with GeneChip Human Gene ST Array (Affimetrix, Santa Clara, CA). The detailed procedure has been described previously (11).

Inhibitor and RNAi studies. SKOV/ip-Fumock and SKOV/ip-FuEP2/Ex 2 cells $\left(5 \times 10^{3}\right.$ cells/well) were plated on 96-well microplates and preincubated overnight at $37^{\circ} \mathrm{C}$. The next day, the cells were treated for $24 \mathrm{~h}$ with BB-94 (a broad MMP inhibitor whose spectrum includes for MMP-7; Santa Cruz Biotechnology), 4-(2-aminoethyl)benzenesulfonyl fluoride hydrochloride (AEBSF, a protease inhibitor whose spectrum includes for TMPRSS4; Tokyo Chemical Industry, Tokyo, Japan), and $\alpha$-naphthoflavone (ANF, an inhibitor of CYP1A and CYP1B; Wako Chemicals, Osaka, Japan) and the number of viable cells determined by the MTT method.

SKOV/ip-Fumock and SKOV/ip-FuEP2/Ex 2 cells $\left(1 \times 10^{5}\right.$ cells/well) were seeded on 6 -well plate and preincubated overnight at $37^{\circ} \mathrm{C}$. The next day, the cells were transfected with negative universal control siRNA (Invitrogen), MMP-7 siRNA (ID SASI_Hs01_00056547; Sigma, St. Louis, MO), TMPRSS4 siRNA (ID SASI_Hs02_00317345; Sigma), and CYP1B1 siRNA (ID SASI_Hs01_00020012; Sigma) with Lipofectamine RNAiMAX (Invitrogen) according to the manufacturer's protocol. After transfection, cells $\left(5 \times 10^{4}\right.$ cells/well) were placed on 6-well plates and incubated for $48 \mathrm{~h}$. The number of viable cells was determined by the trypan blue exclusion method. In parallel, total RNAs from surplus cells were isolated and RT-PCR analysis conducted to confirm their knockdown effects.

In vivo AEBSF treatment. SKOV/ip-FuEP2/Ex2 cells $\left(2 \times 10^{6}\right.$ cells/mouse) were injected into the peritoneal cavity of nude mice $(n=30)$ using a 30-gauge syringe. Starting the next day, mice were treated daily with intraperitoneal saline $(n=10)$, $5 \mathrm{mg} / \mathrm{kg} \operatorname{AEBSF}(\mathrm{n}=10)$ or $20 \mathrm{mg} / \mathrm{kg} \operatorname{AEBSF}(\mathrm{n}=10)$. Treatment continued for 5 days, followed by a 2-day withdrawal period. Mice were weighed every week. After 4 week of observation, the mice were sacrificed and all disseminated tumors in the peritoneal cavity were collected. Tumor lesions were weighed and fixed in $10 \%$ phosphate-buffered formaldehyde for histological analyses.

Statistical analysis. A two-tailed Mann-Whitney U test was employed for comparison of volume of ascites and tumor weights. A two-tailed Student's t-test was employed for comparison of all other data. In all cases, $\mathrm{P}<0.05$ was considered significant.

\section{Results}

Characteristics of SKOV/ip-FuEP2/Ex2 cells. RT-PCR analysis revealed that the SKOV/ip-FuEP2/Ex 2 cells expressed FuEP2/Ex2 mRNA. The mobility shift in SKOV/ip-FuEP2/Ex2 at which we amplified IL2ss-hIgG mRNA was due to the inserted FuEP2/Ex2 cDNA sequence (69 bp). ELISA analysis revealed that successful secretion of $\mathrm{IgG}$-fused proteins into $\mathrm{CM}$ occurred in both cell lines. The concentrations were calculated to be $61.2 \pm 3.3 \mathrm{ng} / \mathrm{ml}$ in SKOV/ip-Fumock cells and $77.4 \pm 1.2 \mathrm{ng} / \mathrm{ml}$ in SKOV/ip-FuEP2/Ex2 cells, respectively (Fig. 1A). The growth rate under normal conditions was next examined using the MTT method and found to be similar in the two cell types 24,48 and $72 \mathrm{~h}$ after starting the experiment (Fig. 1B). RT-PCR analysis also revealed similar expression levels of EP1, EP2, EP3, EP4, mPGES and cPGES mRNA in the two cell lines. In contrast, levels of COX-1 and COX-2 mRNA were lower in SKOV/ip-FuEP2/Ex2 cells. The 
A

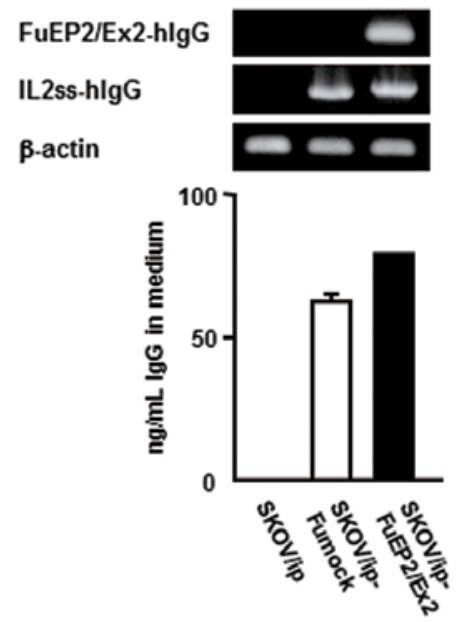

B

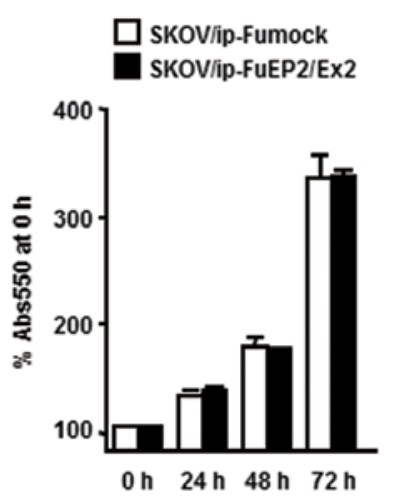

C

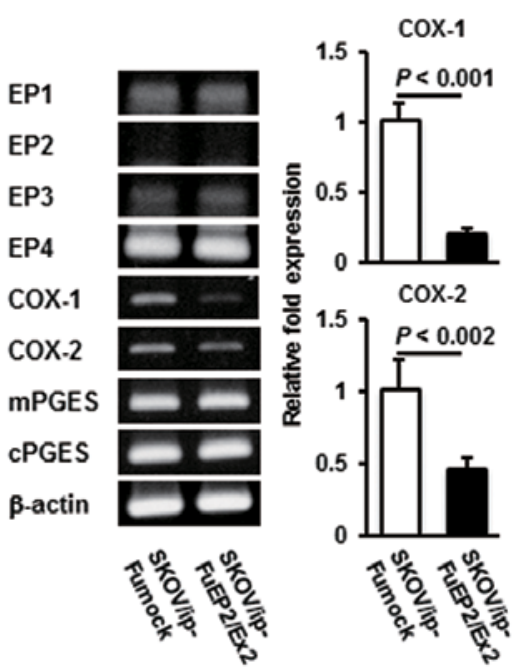

Figure 1. Characteristics of SKOV/ip-FuEP2/Ex2. (A) The mammalian expression vector pFUSE-hFc2 and pFUSE-FuEP2/Ex2 were introduced into SKOV3/ip cells and stable transfectants were established. mRNA expression and protein secretion of the hIgG Fc fragment and FuEP2/Ex2-fused hIgG Fc were confirmed by RT-PCR (upper panel) and ELISA (bottom panel). (B) SKOV3/ip-Fumock and SKOV3/ip-FuEP2/Ex2 cells were incubated in complete medium for 24, 48 and $72 \mathrm{~h}$, and cell proliferation was measured by the MTT assay. The $\mathrm{A}_{550}$ value at $0 \mathrm{~h}$ was assigned as $100 \%$ and the relative percentages at 24,48 and $72 \mathrm{~h}$ are shown. Columns, mean percentages $(n=6)$ of three independent experiments; bars, SD. (C) Expression of EP1-EP4, COX-1, COX-2, mPGES and cPGES mRNA were detected in SKOV3/ip-Fumock and SKOV3/ip-FuEP2/Ex2 cells.

qRT-PCR analysis showed that an $80 \%$ decrease in COX-1 mRNA and a 55\% decrease in COX-2 mRNA were observed in SKOV/ip-FuEP2/Ex2 cells compared to SKOV/ip-Fumock cells (Fig. 1C).

Effect of secreted FuEP2/Ex2 in the peritoneal metastasis model. We intraperitoneally injected SKOV/ip-Fumock and SKOV/ip-FuEP2/Ex2 cells and observed for 28 days. The average body weight of SKOV/ip-Fumock-injected mice began to decrease 21 days after injection, unlike that of SKOV/ip-FuEP2/Ex2-injected mice $(18.2 \pm 1.8 \mathrm{~g}$ in SKOV/ip-Fumock-injected mice vs $19.6 \pm 1.4 \mathrm{~g}$ in $\mathrm{SKOV} / \mathrm{ip}-F u E P 2 / E x 2$-injected mice; $\mathrm{P}=0.058)$. At 28 days, the average body weight of SKOV/ip-Fumock-injected mice had increased because of the accumulation of ascites. Macroscopically, no SKOV/ip-FuEP2/Ex2-injected mice were found to bear ascites (Fig. 2A). As shown in Fig. 2B, SKOV/ip-Fumock-injected mice had large tumors and hemorrhagic ascites. The tumors in SKOV/ip-FuEP2/Ex2-injected mice were smaller: volume of hemorrhagic ascites and mean tumor weight were significantly lower (Fig. 2B). Sections from tumor tissue were also subjected to histological analyses. Like typical tumor xenografts, both tumors were undifferentiated with sarcoma-like findings. Immunohistochemical staining for $\mathrm{hIgG} \mathrm{Fc}$ revealed that both tumors preserved expression of hIgG-derived product (Fig. 2C). We also quantified $\mathrm{Ki}-67-$ labeling indices. The percentage of Ki-67-positive cells did not differ significantly between the two cell lines (Fig. 2C).

We next used ELISA to measure levels of VEGF, IL-6, IL-8 and CXCL1 in CMs and immunohistochemical staining to quantify the number of $\mathrm{mCD} 31$ (as index for microvessel density)-, mCD68 (for total macrophage)- and mCD163 (for M2 macrophage)-positive cells in tumor lesions. ELISA analyses revealed to be significantly lower levels of VEGF, IL-6, IL-8 and CXCL1 in SKOV/ip-FuEP2/Ex2 CM than in SKOV/ip-Fumock
CM (Fig. 3A). As shown in Fig. 3B, the numbers of CD31- and CD163-positive cells were significantly lower in SKOV/ip-FuEP2/ Ex2-derived tumors than in SKOV/ip-Fumock-derived tumors, whereas the number of CD68-positive cells did not differ significantly between the two cell lines.

Determination of combinatorial target with FuEP2/Ex2. By using cDNA microarray analysis, we examined differential gene expression between SKOV/ip-Fumock-derived and SKOV/ip-FuEP2/Ex2-derived tumors. Setting the cut-off value of fold change as $>4.0$ and $<0.25$, respectively, revealed 6 upregulated genes and 8 downregulated genes in the SKOV/ ip-FuEP2/Ex2-derived tumor (Table II). Three upregulated genes encoded enzymes, namely MMP-7 (4.471-fold), TMPRSS4 (4.348-fold) and CYP1B1 (4.000-fold), each of which has previously been shown to be overexpressed in several types of cancer (12-16). To validate the result of cDNA microarray, MMP-7, TMPRSS4 and CYP1B1 mRNA from tumor tissue was detected by RT-PCR. All mRNA levels in SKOV/ip-FuEP2/Ex2-derived tumors appeared to be upregulated compared with those in SKOV/ip-Fumock-derived tumors (Fig. 4).

Effect of inhibition and knockdown of MMP-7, TMPRSS4 and CYPIB1 on growth of ovarian cancer cells. To examine whether MMP-7, TMPRSS4 or CYP1B1 provides a possible target in combination with EP-mediated signaling inhibition, we tested the effects of inhibition and knockdown to their molecules in SKOV/ip-Fumock and SKOV/ip-FuEP2/Ex2 cells. First, BB-94 (inhibitor for MMP-7), AEBSF (for TMPRSS4) and ANF (for CYP1B1) were used to conduct a toxicity assay. BB-94 treatment did not affect cell viability of SKOV/ip-Fumock cells at any dose level; in SKOV/ip-FuEP2/Ex2 cells, significant reduction of cell viability was found in the highest dose group only (Fig. 5A). Comparing SKOV/ip-Fumock and SKOV/ip-FuEP2/Ex2 cells 

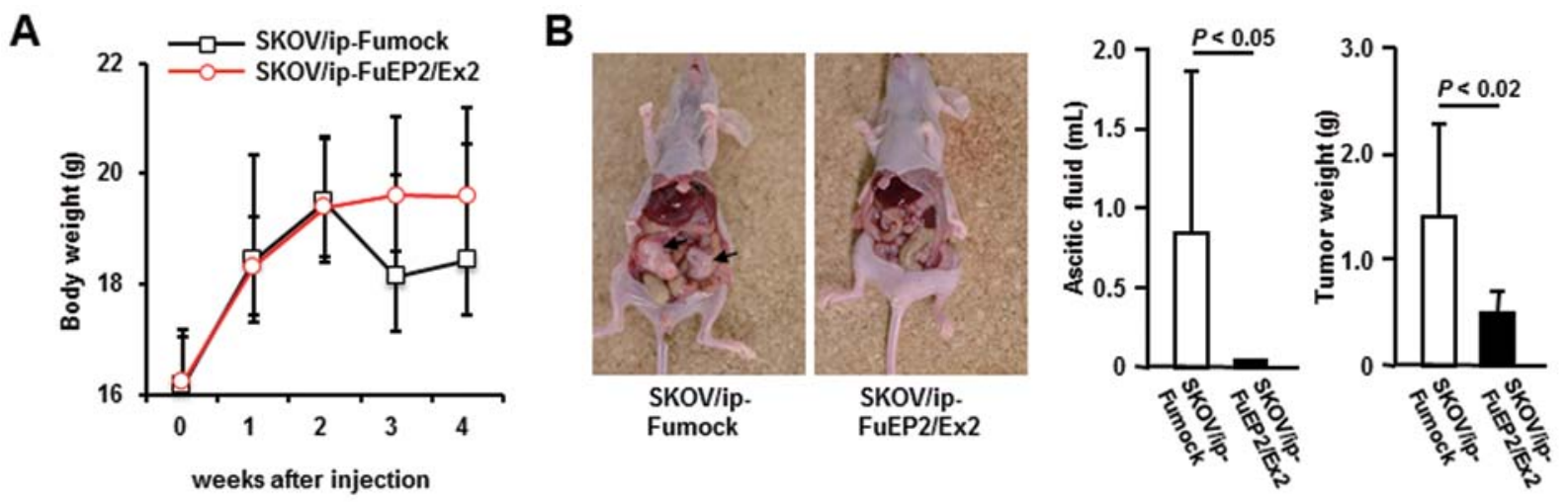

C
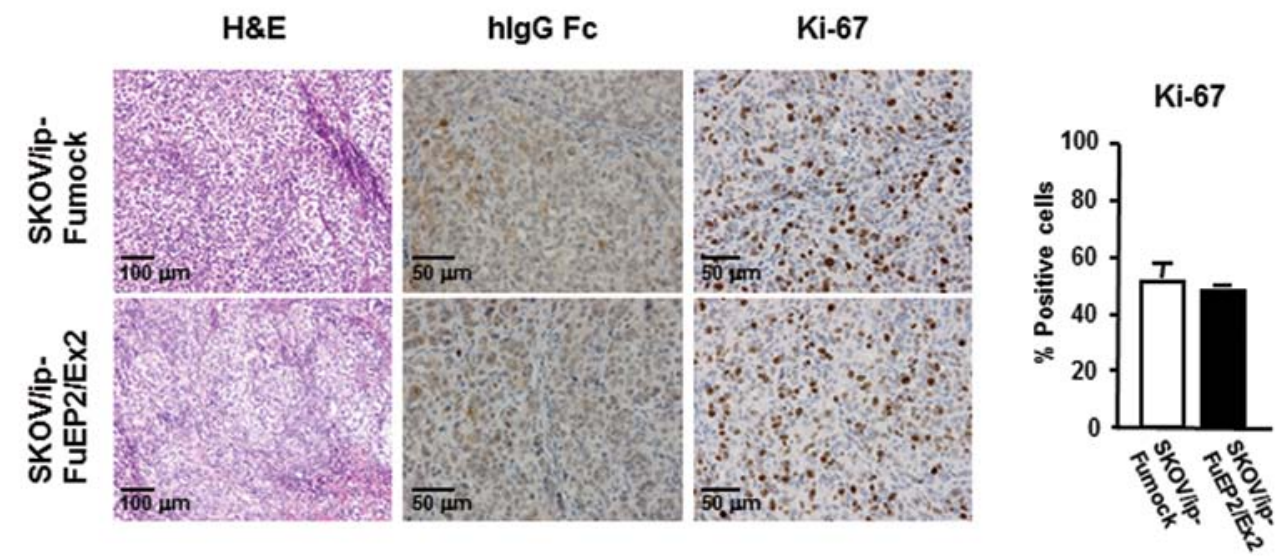

Figure 2. Secreted FuEP2/Ex2 suppresses tumor growth and ascites formation in the peritoneal metastasis model of nude mice. (A) SKOV3/ip-Fumock and SKOV3/ip-FuEP2/Ex2 cells ( $2 \times 10^{6}$ cells/mouse) were intraperitoneally injected into female nude mice. At days $0,7,14,21$ and 28 after injection, SKOV3/ip-Fumock-injected and SKOV3/ip-FuEP2/Ex2-injected mice were weighed. Bars, SD. (B) Macroscopic findings showed an apparent reduction of tumor growth in SKOV3/ip-FuEP2/Ex2-injected mice. Arrows, large tumor lesion. Further, volumes of ascites and tumor weight were measured. Columns, mean; bars, SD. (C) H\&E staining and immunohistochemical staining of hIgG Fc and Ki-67 in tumor lesions. Calculation of the percentage of Ki-67-positive cells was performed. Columns, mean; bars, SD.

Table II. Up- and downregulated genes in tumor lesions by cDNA microarray analysis.

Accession no.

Gene name

Fold change

Upregulated genes in SKOV/ip-FuEP2/Ex2-derived tumor

NM_015068 Paternally expressed 10 (PEG10), transcript variant 1, mRNA

BC021276 Immunoglobulin heavy constant delta, mRNA (cDNA clone IMAGE:4855067)

NM_002423

Matrix metallopeptidase 7 (matrilysin, uterine) (MMP7), mRNA

4.471

NM_019894

Transmembrane protease, serine 4 (TMPRSS4), transcript variant 1 , mRNA

4.348

AB001736

mRNA for scFv collagenase IV antibody, complete cds

4.194

NM_000104 Cytochrome P450, family 1, subfamily B, polypeptide 1 (CYP1B1), mRNA

4.000

Downregulated genes in SKOV/ip-FuEP2/Ex2-derived tumor

NM_001150 Alanyl (membrane) aminopeptidase (ANPEP), mRNA

NM_012082

Zinc finger protein, multitype 2 (ZFPM2), mRNA

0.123

NM_012206

Hepatitis A virus cellular receptor 1 (HAVCR1), transcript variant 1, mRNA

0.149

NM_002364

Melanoma antigen family B, 2 (MAGEB2), mRNA

0.173

NM_020873

Leucine rich repeat neuronal 1 (LRRN1), mRNA

0.213

NM_17586

Transmembrane and tetratricopeptide repeat containing 1 (TMTC1), transcript variant 2, mRNA

0.225

NM_003480

Microfibrillar associated protein 5 (MFAP5), mRNA

0.233

NM_002578

p21 protein (Cdc42/Rac)-activated kinase 3 (PAK3), transcript variant 2, mRNA

0.252 
A
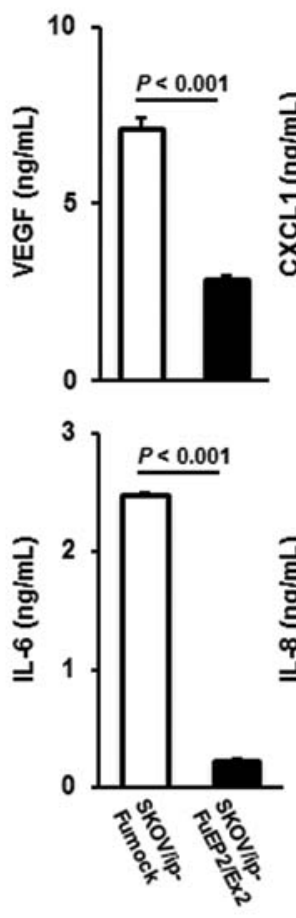
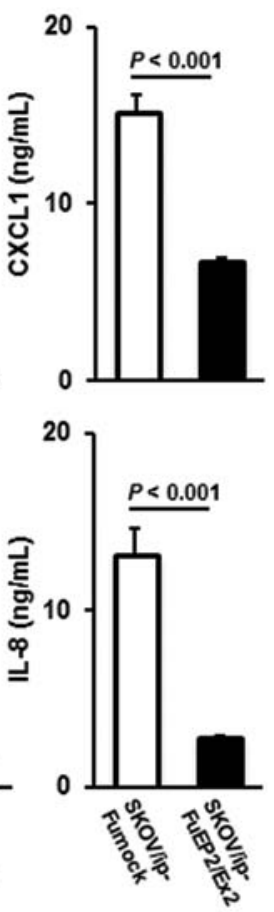

B
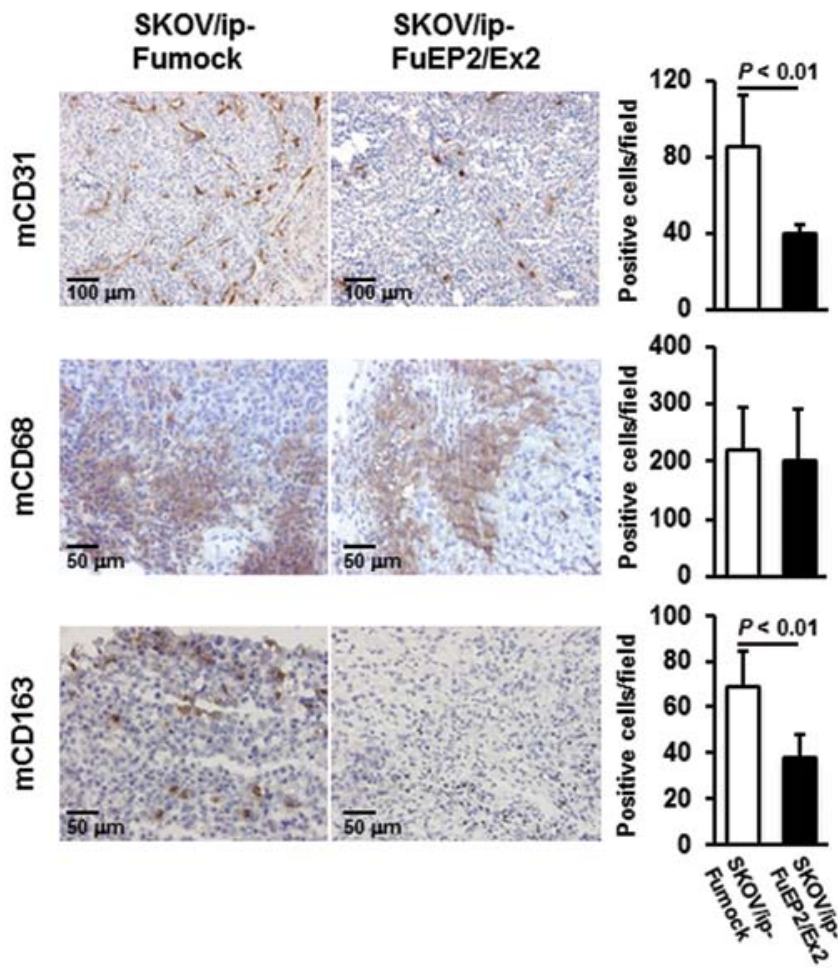

Figure 3. Effect of FuEP2/Ex2 on the production of cytokines and chemokines, tumor angiogenesis, and differentiation to M2 macropharge. (A) SKOV/ip-Fumock and SKOV/ip-FuEP2/Ex2 cells were cultured (4x10 cells/ml for $48 \mathrm{~h}$ ) and the amounts of VEGF, IL-6, IL-8 and CXCL1 in CM were determined by ELISA. Columns, mean $(n=4)$ of three independent experiments; bars, SD. (B) Immunohistochemical staining of mouse CD31, CD68, and CD163 in tumor lesions. The percentages of microvessel densities, CD68-positive cells and CD163-positive cells were calculated. Columns, mean; bars, SD.

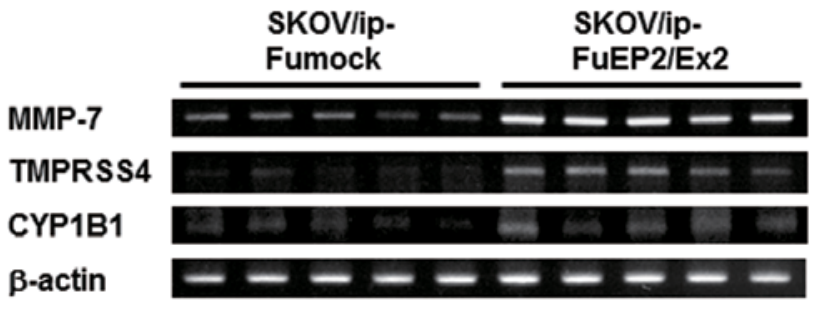

Figure 4. Validation for the cDNA microarray results. Total RNAs from tumor tissue were isolated using an RNeasy Mini kit and then aliquots of these total RNAs (1 $\mu \mathrm{g} / \mathrm{sample})$ were subjected to RT-PCR. The amplicons were hMMP-7, hTMPRSS4, hCYP1B1 and $\beta$-actin. The primer sequences are listed in Table I and $\beta$-actin was amplified as an internal standard.

revealed cell viabilities of all treated groups to be significantly lower in the SKOV/ip-FuEP2/Ex2 than the SKOV/ip-Fumock cell line. Treatment with AEBSF caused dose-dependent reduction of cell viability in both cell lines (Fig. 5A). At the highest dose, cell viability was also significant lower in SKOV/ip-FuEP2/Ex2 than in SKOV/ip-Fumock cells. Treatment with ANF did not affect viability of SKOV/ip-Fumock cells at any dose (Fig. 5A) and significant reduction of SKOV/ip-FuEP2/Ex2 cell viability was found in only the highest dose group (Fig. 5A). As observed with AEBSF treatment, viability of SKOV/ip-FuEP2/Ex2 cells was also significant lower than SKOV/ip-Fumock cells at the highest dose.
Next, we conducted a knockdown assay using specific siRNA for MMP-7, TMPRSS4 and CYP1B1. RT-PCR analysis revealed that all kinds of siRNA worked effectively and specifically in both SKOV/ip-Fumock and SKOV/ip-FuEP2/Ex 2 cells (Fig. 5B). Using these knocked down cells, we assessed growth activity by the trypan blue-exclusion method. As shown in Fig. 5B, there were significantly fewer SKOV/ip-FuEP2/Ex2 cells than SKOV/ip-Fumock cells following treatment with TMPRSS4 siRNA.

Effect of AEBSF treatment in a SKOV/ip-FuEP2/Ex2-based peritoneal metastasis model. Based on our in vitro results, inhibition of TMPRSS4 is a likely candidate to provide synergistic suppression of cancer cell growth in vivo under depletion of EP-mediated signaling. To test this proposal, we intraperitoneally injected SKOV/ip-FuEP2/Ex 2 cells and administered saline or AEBSF ( 5 or $20 \mathrm{mg} / \mathrm{kg}$ ) to the nude mice. At experiment end-point, average body weight in the $20 \mathrm{mg} / \mathrm{kg}$ AEBSF-treated group was slightly lower than in the other two groups, but the difference was not significant $(21.2 \pm 1.06 \mathrm{~g}$ saline, $21.0 \pm 0.87 \mathrm{~g}$ $5 \mathrm{mg} / \mathrm{kg}$ AEBSF, 20.2 $\pm 1.61 \mathrm{~g} 20 \mathrm{mg} / \mathrm{kg}$ AEBSF, respectively; data not shown). Tumor weight decreased dose-dependently with AEBSF (Fig. 6A). Using paraffin-embedded sections of tumor lesion, Ki-67-labeling indices were quantified and TUNEL-positive apoptotic cells were counted. The percentage of Ki-67-positive cells was significantly lower in AEBSFtreated groups than the saline-treated group, while the numbers of TUNEL-positive cells did not differ significantly (Fig. 6B). 
A

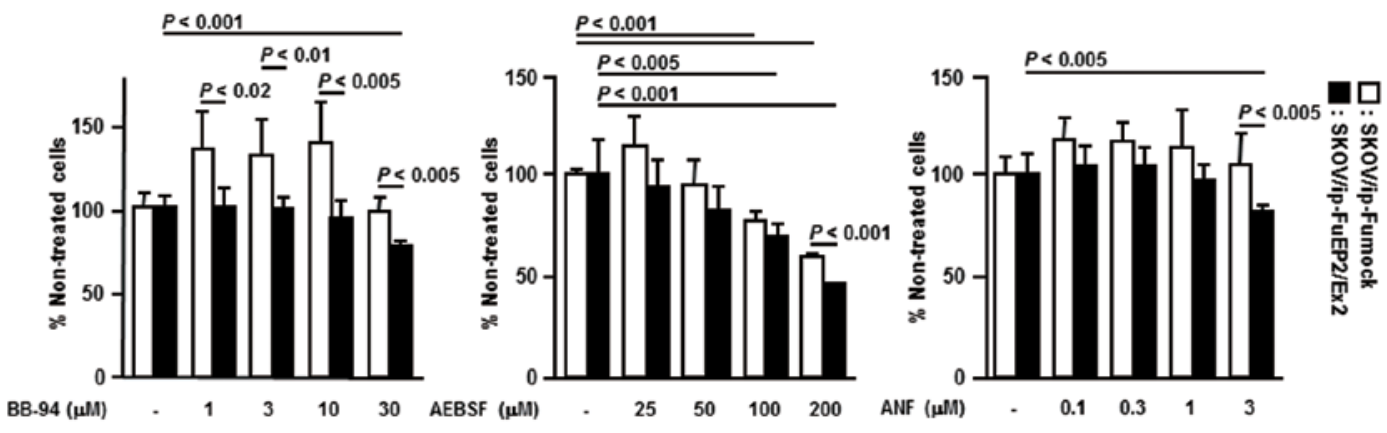

B
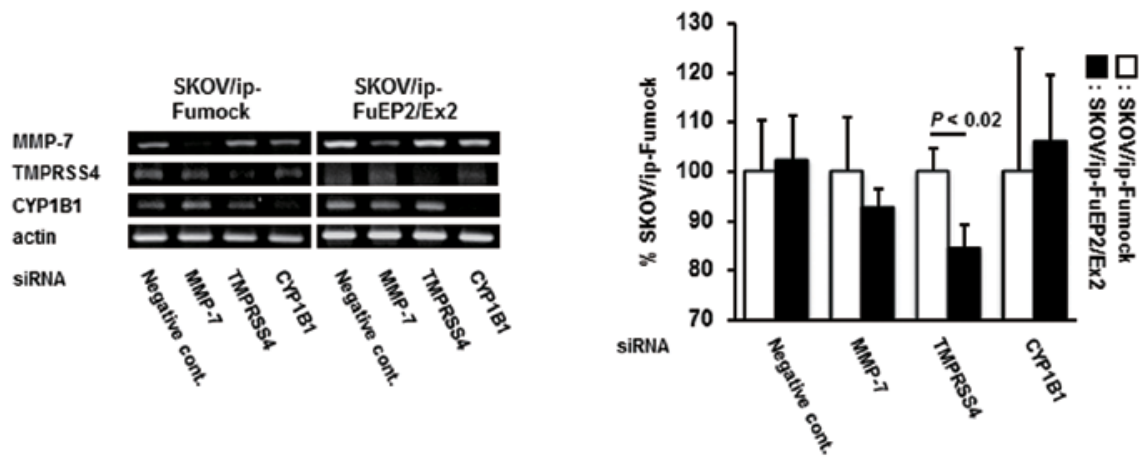

Figure 5. Effects of dual targeting of EP receptor-mediated cell signaling by FuEP2/Ex2 with MMP-7, TMPRSS4 and CYP1B1 in vitro. (A) SKOV/ip-Fumock and SKOV/ip-FuEP2/Ex 2 cells were treated with four doses of BB-94, AEBSF and ANF for $24 \mathrm{~h}$ and cell viability was measured by the MTT assay. The $\mathrm{A}_{550}$ value for untreated cells was assigned as $100 \%$ and the relative percentages of treated cells are shown. Columns, mean percentages $(\mathrm{n}=6)$ of three independent experiments; bars, SD. (B) Knockdown by specific siRNA for MMP-7, TMPRSS4 and CYP1B1 was performed in SKOV/ip-Fumock and SKOV/ip-FuEP2/Ex2 cells. RT-PCR analyses were conducted to confirm whether knockdown was successful (left). The growths of knocked down cells were calculated by counting cells with the trypan blue exclusion method (right). Cell numbers in SKOV/ip-Fumock were assigned as 100\% and the relative percentages in SKOV/ip -FuEP2/Ex2 are shown. Columns, mean $(\mathrm{n}=3)$ of three independent experiments; bars, SD.

A

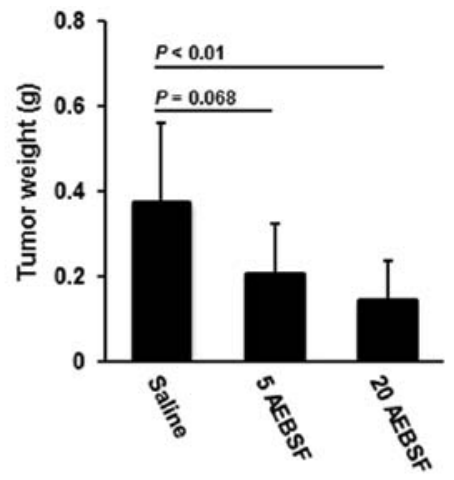

B
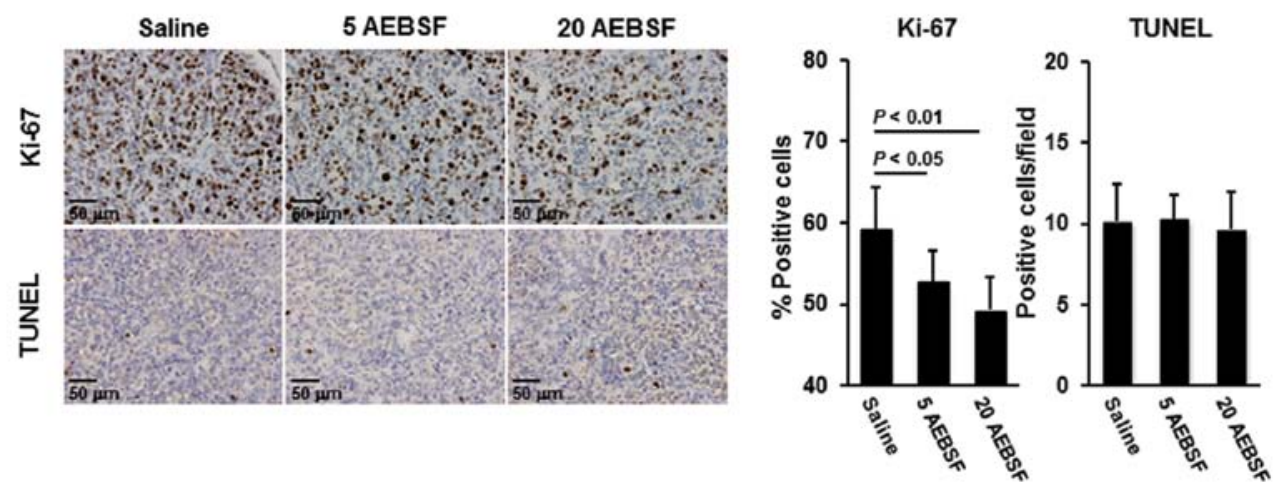

Figure 6. Inhibition of TMPRSS4 by AEBSF synergistically suppresses cancer cell growth in the SKOV/ip-FuEP2/Ex2-based peritoneal metastasis model of nude mice. (A) Representative picture of whole tumor lesions of saline-, $5 \mathrm{mg} / \mathrm{kg} \mathrm{AEBSF}$ - and $20 \mathrm{mg} / \mathrm{kg}$ AEBSF-treated mice (left). Average tumor weights of the three groups were calculated (right). Columns, mean; bars, SD. (B) Immnunohistochemical staining of Ki-67 and TUNEL staining were performed. The percentages of Ki-67-positive cells and TUNEL-positive cells were calculated. Columns, mean; bars, SD. 


\section{Discussion}

Cellular signaling via the $\mathrm{PGE}_{2}$-EP receptor is involved in the growth, progression and metastasis of ovarian cancer as well as many other cancer types $(8,17,18)$. Indeed, a strategy that blocks production of $\mathrm{PGE}_{2}$ or EP receptor-mediated signaling showed a reduction in cancer cell growth in vitro and in vivo (19-21). Heterozygous deletion of the COX-2 gene also reduced growth of ovarian cancer (22). These reports suggest that cellular signaling via the $\mathrm{PGE}_{2}-\mathrm{EP}$ receptor can be a target for therapy of ovarian cancer and its metastasis. Therefore, we established FuEP2/Ex2-expressing ovarian cancer cells and attempted to evaluate the suppressive effect of FuEP2/Ex2, which functions as a decoy receptor to $\mathrm{PGE}_{2}$, on tumor growth in a peritoneal metastasis model.

The FuEP2/Ex2-expressing cells, SKOV/ip-FuEP2/Ex2, grew at a similar rate to vector-control cells. Surveying the expression status of EP receptors and enzymes involved in producing $\mathrm{PGE}_{2}$ revealed that COX-1 and COX-2 mRNA were downregulated in SKOV/ip-FuEP2/Ex2 cells. Exogenous $\mathrm{PGE}_{2}$ stimulates expression of COX-1 and COX-2 mRNA $(23,24)$, so this result indicates that FuEP2/Ex 2 cuts off the positive feedback loop of the $\mathrm{PGE}_{2}-\mathrm{COX}$ axis. Similar growth rate may be due to compensation by other factors under culture conditions. An in vivo experiment revealed that FuEP2/Ex2 inhibits ovarian tumor growth with complete disappearance of ascetic fluid and decreased tumor weight. In SKOV/ip-FuEP2/Ex2 cells, production of VEGF, CXCL1, IL-6 and IL-8, which contributes to the generation of ascitic fluid and enhancement of tumor growth in ovarian cancer (25-28), is suppressed under in vitro conditions. These factors can also be regulated by $\mathrm{PGE}_{2}$ (29-32). Our data suggest that blockade of EP receptor-mediated cell signaling by FuEP2/Ex2 also influences the production of these factors and that these reductions may cause the disappearance of ascetic fluid and the decreased tumor weight. Immunohistochemical staining showed decreased microvessel density and M2 macrophages in SKOV/ip-FuEP2/Ex2-derived tumors. M2 macrophage is an activator of tumor growth and a suppressor of tumor immunity, and $\mathrm{PGE}_{2}, \mathrm{VEGF}$ and IL-6 are known to be an inducer of differentiation into M2 macrophages (33). These results further strengthen the suggestion that FuEP2/Ex2 can suppress angiogenesis, which is one of the most important events in intraperitoneal metastasis of ovarian cancer and that its growth suppression is also associated with blocking of differentiation into M2 macrophages by secreted FuEP2/Ex2.

cDNA microarray analysis revealed that several genes are up- or downregulated in the SKOV/ip-FuEP2/Ex2-derived tumor lesion. We focused on upregulated genes because of their possible role in cell survival under depletion of EP receptormediated cell signaling. Of the upregulated genes, we chose MMP-7, TMPRSS4 and CYP1B1, and analyzed whether interference to these genes synergistically affects neutralization of EP receptor-mediated cell signaling by FuEP2/Ex 2 . The reason why we chose these three genes is that they have enzymatic function, allowing use of their inhibitors for further analysis. Toxicity assays using their inhibitors showed the TMPRSS4 inhibitor AEBSF to have toxic effect on both SKOV/ip-Fumock and SKOV/ip-FuEP2/Ex2 cells. At the highest dose, its toxicity was greater towards SKOV/ip-FuEP2/Ex2 than SKOV/
ip-Fumock cells. Both the MMP-7 inhibitor BB-94 and the CYP1B1 inhibitor ANF had weaker effects than AEBSF in both cell lines. Experiments using siRNA showed that the number of SKOV/ip-FuEP2/Ex2 cells was lower than SKOV/ip-Fumock cells when treated with TMPRSS4 siRNA. These results suggest that TMPRSS4 inhibition has the potential to enhance the effect of FuEP2/Ex2. However, we were concerned that the toxic and suppressive effects of targeting TMPRSS4 are not so obvious in vitro. Therefore, we checked the expression status of this gene by performing another cDNA microarray analysis under in vitro conditions. This attempt revealed that the degree of upregulation in SKOV/ip-FuEP2/Ex2 was apparently decreased (1.34-fold; data not shown). This indicates that upregulation of these genes is due to interactions with cells from the host rather than FuEP2/Ex2 itself. Therefore, we continued to analyze the effect of AEBSF in our SKOV/ip-FuEP2/Ex2-based peritoneal metastasis model.

Treatment of AEBSF further suppressed tumor growth in the SKOV/ip-FuEP2/Ex2-based peritoneal metastasis model. This suppression was dose-dependent without toxicity. Immunohistochemical analyses revealed that this suppression is associated with proliferative status of cancer cells rather than apoptotic induction, unlike the difference between SKOV/ip-Fumock and SKOV/ip-FuEP2/Ex2 cells. In contrast, single administration of AEBSF did not affect cancer cell growth and ascites formation (data not shown). These results may suggest that AEBSF could have an effect in the presence of FuEP2/Ex2. We also tested the effect of single or combination treatment of AEBSF and meloxicam, a selective COX-2 inhibitor; however, no suppressive effects on cancer cell growth and ascites formation were observed (data not shown). This indicates that some prostanoids derived from COXs suppress ovarian tumor growth and that specific inhibition of EP receptor-mediated cell signaling is a valid therapeutic strategy for peritoneal metastasis of ovarian cancer. TMPRSS4 promotes tumor growth, invasion, metastasis, and epithelialmesenchymal transition $(15,34)$. Considering the function of TMPRSS4 in cancer cells, our data showing inhibition of cancer cell growth in SKOV/ip-FuEP2/Ex2-based peritoneal metastasis model are reasonable, and the ineffectiveness of AEBSF alone or in combination with meloxicam may be a result of the expression level of TMPRSS4 being insufficient to suppress cancer cell growth.

Our above results strongly suggest potential targets for treatment of ovarian cancer metastasis that is positive for EP (especially EP2 and EP4) and TMPRSS4. At present, there have been few reported clinical studies using EP antagonists $(35,36)$ and no specific inhibitor for TMPRSS4 has yet been developed. If a potent, specific, and safe way to attack these targets is exploited, it may provide a promising therapeutic strategy for the repression of ovarian cancer metastasis by combining existing agents for ovarian cancer therapy.

\section{Acknowledgements}

The authors thank Megumi Kume and Hitomi Umemoto for technical assistance. This study was supported in part by The Ministry of Education, Culture, Sports, Science and Technology of Japan, Grant-in-Aid for Young Scientists (B), nos. 22700920 and 24701026 (T.T.). 


\section{References}

1. Jemal A, Bray F, Center MM, Ferlay J, Ward E and Forman D Global cancer statistics. CA Cancer J Clin 61: 69-90, 2011.

2. Yokoyama Y, Sakamoto T, Sato S and Saito Y: Evaluation of cytoreductive surgery with pelvic and paraaortic lymphadenectomy and intermittent cisplatin-based combination chemotherapy for improvement of long-term survival in ovarian cancer. Eur J Gynaecol Oncol 20: 361-366, 1999.

3. Wang D and Dubois RN: Prostaglandins and cancer. Gut 55: $115-122,2006$

4. Freedman RS, Wang E, Voiculescu S, et al: Comparative analysis of peritoneum and tumor eicosanoids and pathways in advanced ovarian cancer. Clin Cancer Res 13: 5736-5744, 2007.

5. Shigemasa K, Tian X, Gu L, Shiroyama Y, Nagai N and Ohama K: Expression of cyclooxygenase-2 and its relationship to $\mathrm{p} 53$ accumulation in ovarian adenocarcinomas. Int $\mathrm{J}$ Oncol 22: 99-105, 2003.

6. Daikoku T, Tranguch S, Trofimova IN, et al: Cyclooxygenase-1 is overexpressed in multiple genetically engineered mouse models of epithelial ovarian cancer. Cancer Res 66: 2527-2531, 2006.

7. Coleman RA, Smith WL and Narumiya S: International union pharmacology classification of prostanoid receptors: properties distribution and structure of the receptors and their subtypes. Pharmacol Rev 46: 205-229, 1994.

8. Rask K, Zhu Y, Wang W, Hedin L and Sundfeldt K: Ovarian epithelial cancer: a role to PGE2-synthesis and signaling in malignant transformation and progression. Mol Cancer 5: 62, 2006.

9. Takahashi T, Uehara H, Bando Y and Izumi K: Soluble EP2 neutralizes prostaglandin $\mathrm{E}_{2}$-induced cell signaling and inhibits osteolytic tumor growth. Mol Cancer Ther 7: 2807-2816, 2008

10. Takahahshi T, Ogawa H, Izumi K and Uehara H: The soluble EP2 receptor FuEP2/Ex2 suppresses endometrial cancer cell growth in an orthotopic xenograft model in nude mice. Cancer Lett 306: 67-75, 2011.

11. Shiirevnyamba A, Takahashi T, Shan H, et al: Enhancement of osteoclastogenic activity in osteolytic prostate cancer cells by physical contact with osteoblasts. Br J Cancer 104: 505-513, 2011.

12. Mori M, Barnard GF, Mimori K, Ueo H, Akiyoshi T and Sugimachi K: Overexpression of matrix metalloproteinase-7 mRNA in human colon carcinomas. Cancer 75: 1516-1519, 1995.

13. Honda M, Mori M, Ueo H, Sugimachi K and Akiyoshi T: Matrix metalloproteinase-7 expression in gastric carcinoma. Gut 39: 444-448, 1996.

14. Kebebew E, Peng M, Reiff E, Duh QY, Clark OH and McMillan A: ECM1 and TMPRSS4 are diagnostic markers of malignant thyroid neoplasms and improve the accuracy of fine needle aspiration biopsy. Ann Surg 242: 353-361, 2005.

15. Kim S, Kang HY, Nam EH, et al: TMPRSS4 induces invasion and epithelial-mesenchymal transition through upregulation of integrin alpha5 and its signaling pathways. Carcinogenesis 31: 597-606, 2010

16. McFadyen MC, Cruickshank ME, Miller ID, et al: Cytochrome P450 CYP1B1 over-expression in primary and metastatic ovarian cancer. Br J Cancer 85: 242-246, 2001.

17. Heinonen PK and Metsä-Ketelä T: Prostaglandin and thromboxane production in ovarian cancer tissue. Gynecol Obstet Invest 18: 225-229, 1984

18. Schmidt C, Laporte A and De Baetselier P: Differential responsiveness of murine $\mathrm{T}$ lymphomas to local growth and invasion factors may determine metastasis formation in the ovaries. Invasion Metastasis 17: 53-61, 1997.

19. Spinella F, Rosanò L, Di Castro V, Natali PG and Bagnato A Endothelin-1-induced prostaglandin E2-EP2, EP4 signaling regulates vascular endothelial growth factor production and ovarian carcinoma cell invasion. J Biol Chem 279: 46700-46705, 2004.
20. Li W, Ji ZL, Zhuo GC, Xu RJ, Wang J and Jiang HR: Effects of selective cyclooxygenase-1 inhibitor in SKOV-3 ovarian carcinoma xenograft-bearing mice. Med Oncol 27: 98-104, 2010.

21. Uddin S, Ahmed M, Hussain A, et al: Cyclooxygenase-2 inhibition inhibits PI3K/AKT kinase activity in epithelial ovarian cancer. Int J Cancer 126: 382-394, 2010.

22. Yang WL, Cai KQ, Smedberg JL, et al: A reduction of cyclooxygenase 2 gene dosage counters the ovarian morphological aging and tumor phenotype in Wv mice. Am J Pathol 170: 1325-1336, 2007.

23. Orman B, Reina S, Sterin-Borda L and Borda E: Signaling pathways leading to prostaglandin $\mathrm{E}(2)$ production by rat cerebral frontal cortex. Prostaglandins Leukot Essent Fatty Acids 74: 255-262, 2006.

24. Sales KJ, Grant V and Jabbour HN: Prostaglandin E2 and F2alpha activate the FP receptor and up-regulate cyclooxygenase-2 expression via the cyclic AMP response element. Mol Cell Endocrinol 285: 51-61, 2008.

25. Mesiano S, Ferrara N and Jaffe RB: Role of vascular endothelial growth factor in ovarian cancer: inhibition of ascites formation by immunoneutralization. Am J Pathol 153: 12491256,1998

26. Bolitho C, Hahn MA, Baxter RC and Marsh DJ: The chemokine CXCL1 induces proliferation in epithelial ovarian cancer cells by transactivation of the epidermal growth factor receptor. Endocr Relat Cancer 17: 929-940, 2010.

27. Erroi A, Sironi M, Chiaffarino F, Chen ZG, Mengozzi M and Mantovani A: IL-1 and IL-6 release by tumor-associated macropharges from human ovarian carcinoma. Int J Cancer 44: 795-801, 1989.

28. Yoneda J, Kuniyasu H, Crispens MA, Price JE, Bucana CD and Fidler IJ: Expression of angiogenesis-related genes and progression of human ovarian carcinomas in nude mice. J Natl Cancer Inst 90: 447-454, 1998

29. Wang X and Klein RD: Prostaglandin E2 induces vascular endothelial growth factor secretion in prostate cancer cells through EP2 receptor-mediated cAMP. Mol Carcinog 46: 912-923, 2007

30. Wang D, Wang $\mathrm{H}$, Brown J, et al: CXCL1 induced by prostaglandin E2 promotes angiogenesis in colorectal cancer. J Exp Med 203: 941-951, 2006.

31. Ray A, Sassone-Corsi P and Sehgal PB: A multiple cytokineand second messenger-responsive element in the enhancer of the human interleukin-6 gene: similarities with c-fos gene regulation. Mol Cell Biol 9: 5537-5547, 1989.

32. Caristi S, Piraino G, Cucinotta M, Valenti A, Loddo S and Teti D: Prostaglandin E2 induces interleukin-8 gene transactivation by activating C/EBP homologous protein in human T lymphocytes. J Biol Chem 280: 14433-14442, 2005.

33. Heusinkveld M, de Vos van Steenwijk PJ, Goedemans R, et al: M2 macrophages induced by prostaglandin E2 and IL-6 from cervical carcinoma are switched to activated M1 macrophages by CD4+ Th1 cells. J Immunol 187: 1157-1165, 2011.

34. Jung H, Lee KP, Park SJ, et al: TMPRSS4 promotes invasion, migration and metastasis of human tumor cells by facilitating an epithelial-mesenchymal transition. Oncogene 27: 2635-2647, 2008.

35. Sarkar S, Hobson AR, Hughes A, et al: The prostaglandin E2 receptor-1 (EP-1) mediates acid-induced visceral pain hypersensitivity in humans. Gastroenterology 124: 18-25, 2003.

36. Antonova M, Wienecke T, Maubach K, Thomas E, Olesen J and Ashina M: The pharmacological effect of BGC20-1531, a novel prostanoid EP4 receptor antagonist, in the prostaglandin E2 humanmodel of headache. J Headache Pain 12 551-559, 2011. 